\title{
Decision Support for Life-Cycle Optimization Using Risk Assessment
}

\author{
Maria Marques ${ }^{1}$ and Rui Neves-Silva ${ }^{2}$ \\ ${ }^{1}$ UNINOVA - Instituto de Desenvolvimento de Novas Tecnologias, \\ FCT Campus, 2829-516 Caparica, Portugal \\ Tel.: +351212947832 \\ mcmeuninova.pt \\ ${ }^{2}$ FCT/UNL - Universidade Nova de Lisboa, FCT Campus, 2829-516 \\ Caparica, Portugal \\ rns@fct.unl.pt
}

\begin{abstract}
The key idea of this work, is to use risk assessment to support the user in deciding which service should be used, from a set of services developed to support life-cycle optimization, in a specific situation. The risk of a specific situation affecting an industrial plant, characterized by the symptoms, is estimated from the information stored on the system concerning the probability of occurrence of the consequence and its impact. It is expected that this knowledge grows along the life-cycle of a industrial plant. Then, depending of the knowledge available and on the risk of the situation, the adequate service is suggested for promptly reaction in eliminating the problem or avoiding critical situations. ${ }^{1}$
\end{abstract}

Keywords: Risk Assessment, Decision Support, Life-cycle optimization.

\section{Introduction}

Life Cycle Management (LCM) has been developed as a business approach for managing the total life cycle of products and services. By learning how to more effectively manage this cycle, a company or organisation can uncover a wealth of business, environmental and social value - and make the choice to engage in more sustainable activities and production patterns.

Life Cycle Management is all about making more informed business decisions and chances are that life cycle considerations are already influencing the decisions currently made. Life Cycle Management is simply about helping making these decisions in a more deliberate and systematic way - so a more sustainable production and consumption is possible, and clearly define and measure the business value gained by doing so [1]. Life-cycle management of industrial plants is about taking decisions on a daily basis. What configuration is best, what production pattern, when to replace a part, who should be involved in a maintenance process, etc. On the other side, an

1 This work was partially supported by Commission of European Union within the project InLife (Integrated Ambient Intelligence and Knowledge-Based Services for Optimal LifeCycle Impact of Complex Manufacturing and Assembly Lines), under the Sixth Research Framework Program of the European Union, with the contract NMP2-CT-2005-517018. 
industrial plant can produce, through the available instrumentation, a huge amount of information from where decisions can be supported if well interpreted.

Thus the central problem is on how to combine the existing information so that we can foresee what impact a specific decision will have. Most of the times the information is not structured and it is scattered along the plant making extremely difficult to correlate it and reach a consistent conclusion, i.e. a decision.

In terms of decision theory reaching a conclusion is choosing among a set of alternatives. The parameters involved in this process are treated as subjective judgments, expressing the decision-maker's knowledge, experience, and intuition [2]. Nevertheless, some research using naturalistic methods shows, that in situations with higher time pressure, higher stakes, or increased ambiguities, experts give a more important role to intuitive decision making rather than structured approaches, following recognition primed decision approach to fit a set of indicators into the expert's experience and immediately arrive at a satisfactory course of action without weighing alternatives [3]. Due to the large number of considerations involved in many decisions, computerbased decision support systems have been developed to assist decision makers in considering the implications of various courses of thinking. These systems can help reduce the risk of human errors due to cognitive and temporal limitations [4]. All these aspects become clear when the decision must be made in a noisy environment or in a distressing situation, which are very common conditions in industrial plants.

The work here presented was developed under the scope of the InLife project. One of the objectives of InLife was to develop a system that uses the data coming from different sources along the industrial plant to support decisions for lifecycle management [5]. From the analysis of the received data, and in case there are changes that may cause an increase of the risk level, the system is able to fire adequate services that suggest actions helping the user deal with the situation. Additionally the incoming data is used to compute life-cycle parameters, defined by the user. If any of the defined parameters exceed a predefined threshold, than the same strategy is applied, which will provide support to the life-cycle management.

\section{Contribution to Technological Innovation}

Approach for system development. The system comprises a service platform, which constitutes the user interface. Thus, the existing services will interact with the user presenting the information available in a structured and suitable way. The system supports the user by providing suggestions in terms of the most suitable service to be used in a specific situation, as well as in making a decision considering the relevant information for each case. Accordingly, the system is responsible for analysing the data received, process it and transform it into knowledge that is used to deliver services to the user. The generation/collection of knowledge is based on the use of Ambient Intelligence technology (AmI) [9],[10], Knowledge Management (KM) techniques [11] and the developed service platform [8].

Among the set of services offered three are in the core of the decision support approach: Condition-based Maintenance, Online Remote Maintenance and Diagnostics, and Prevention of Hazardous Situations. They were selected since they are seen as services to be used on a daily basis operation, especially the first two. Thus the level 
of decision involved and the impact those decisions will have is more interesting in terms of life-cycle management then the services used occasionally.

The services main function is to provide support for actions to achieve a specific service goal through recommendations to the human user of the system. These recommendations result from the understanding of the plant operation by the system which is the result of modelling cause effect relations (cause events/actions to consequence effects) achieved by an effective life-cycle parameter monitoring.

These relations are used for the establishment of cases that describe the effects of a certain cause. The collection of these cases is then used to select the best option, from the set of already available, for a specific situation. This is the strategy used to implement the decision support approach based on the utilization of the three above mentioned services.

System Entities. In order to have the system installed and running in several industrial plants there is the need for developing a model, general enough that comprises all the relevant information on plant operation. Nevertheless, and despite the generic character of the model it is also required that it includes several aspects of the operation. Thus the result requires a good balance between simplification of some aspects and the complexity that still is associated to the production process. Since the developed model to support the entire system includes some aspects that are not relevant for the work here described, we will focus on the entities that are vital for the application of the proposed approach.

Fig. 1 shows part of the developed model simply showing the entities that are directly involved on the risk assessment and in support to decision process.

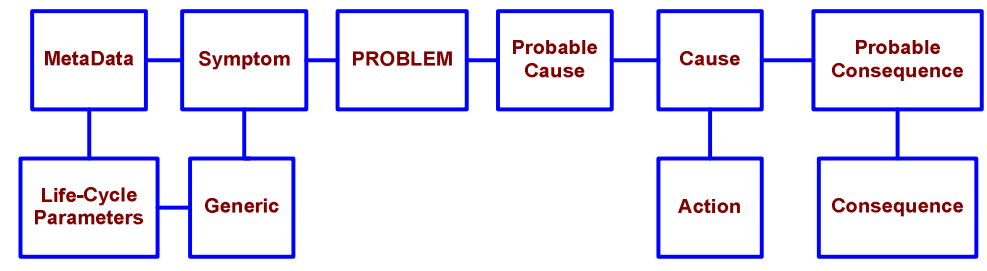

Fig. 1. Simplified developed model for saving data from plant

The entity Problem is the central part of the entire system, since any deviation on the defined normal behaviour of the plant will be stored has an instance of this entity. This entity is connected to Symptom, representing the firing event for the risk assessment process, and to Probable Cause, which is a set of possible causes for the occurred situation (among a list of predefined causes). The Symptom entity makes the connection between the lower level part of the model and the upper one. In the lower level we can find entities to represent the operating parts of the plant like Generic (representing machines involved in production, production processes or product parts) and Life-Cycle Parameters (representing the defined parameters that are seen as relevant for the plant). Additionally, MetaData is used to represent the a priori knowledge related with the plant (e.g. in terms of which Generics are related to which Life-Cycle Parameters so that an abnormal value of these latest generate a Symptom). In the upper part of the model the aim is to store the information 
associated to causes and actions that are related to the problems. Additionally the information on which are the probable causes for a specific problem and the probable consequences for each cause are also stored.

All these entities have several fields that are used to store the associated information, once again, and for the sake of simplicity, here we will only refer the ones that are used in the context of this work. Thus, and in what refers to Problem, the field frequency is used to represent the certainty of knowledge about a problem. The problems with frequency higher than 1 did not occurred, but they were artificially introduced at setup stage and are reference expected problems for each symptom.. Thus, when a similar problem occur the probability of the cause to be the same defined for the problem with higher frequency is higher than for other different problems that only occurred once.

In what concerns Probable Cause the fields probability and risk are used. The first one will store the probability of that cause to be the correct one, whereas the second will store the value of the calculated risk during risk assessment. Note that a Probable Cause is unique but two probable causes can be associated to the same cause. The Probable Consequence has a field to store the probability of a specific cause to induce a specific consequence. Finally, Consequence has a field to store the impact that that consequence will have (if occurs). On the other part of the model we find that Symptom has a field to identify the Generic where the abnormal life-cycle parameter value occurred (given by the connection between Generic, Life-Cycle Parameter and MetaData).

In terms of innovation three aspects are considered as major contributions: (i) the use of AmI and KM to collect and manage the information generated by the plant and its users; (ii) the developed model; and (iii) the use of the information to develop the risk assessment and provide support to decision to the user based on the results.

\section{Risk Assessment}

Risk is a concept that denotes a potential negative impact to an asset or some characteristic of value that may arise from some present process or future event. In everyday usage, "risk" is often used synonymously with "probability" of a loss or threat. Nevertheless, risk should combine the probability of an event occurring with the impact that the event would have and with its different circumstances [12],[13].

Risk assessment is considered as the initial and periodical step in a risk management process. Risk assessment is the determination of quantitative or qualitative value of risk related to a concrete situation and a recognised threat [14]. Thus, risk $\mathrm{R}$ is defined as the result of the product between the probability of occurrence of a specific incident $\mathrm{E}$ and the impact I of that incident (in money or injuries), i.e.

$$
R=P_{o}(E) \times I(E)
$$

If any of the relevant parameters defined for the plant crosses its threshold, the information provided by the system allows the probability of an incident to be estimated. Also, the estimated consequences of the incident allow the estimation of the level of risk that we are dealing with. The functions that describe both incident probability and losses are supposed to be known (even if with some level of uncertainty). This information is collected during set-up phase and during operation using operator knowledge in order to compute the risk. 
Assessment Method. In what concerns InLife the calculated risk is presented in cost units. The values of threshold between risk levels are to be defined by the industrial users since they may not be adequate for all cases [6].

The use of Event Tree Analysis, using Bayesian networks [15], for assessing the probability of occurrence is seen as the most suitable method to achieve the final consequences. An Event Tree starts from an undesired initiator (loss of critical supply, component failure etc) and follows possible further system events through to a series of final consequences. As each new event is considered, a new node on the tree is added with a split of probabilities of taking either branch. The probabilities of a range of 'top events' arising from the initial event can then be seen [16]. The method described corresponds to the so called "Probabilistic Risk Assessment" and as an analytical tool includes the consideration of the following:

- Identification of the combinations of events that, if occur, could lead an undesired event;

- Estimation of the chance of occurrence for each combination; and

- Estimation of the consequences associated with each combination.

For instance, consider that an alarm is fired indicating that there is a deviation on the expected value of "mean-time-between-failure", expressing that this value exceeded some threshold that is considered normal. Assuming that the occurrence of this type of situation is known as generating a specific loss, that must be quantified, and that the probability of incident associated to the fired alarm is also known, than it is possible to calculate the risk associated to this situation.

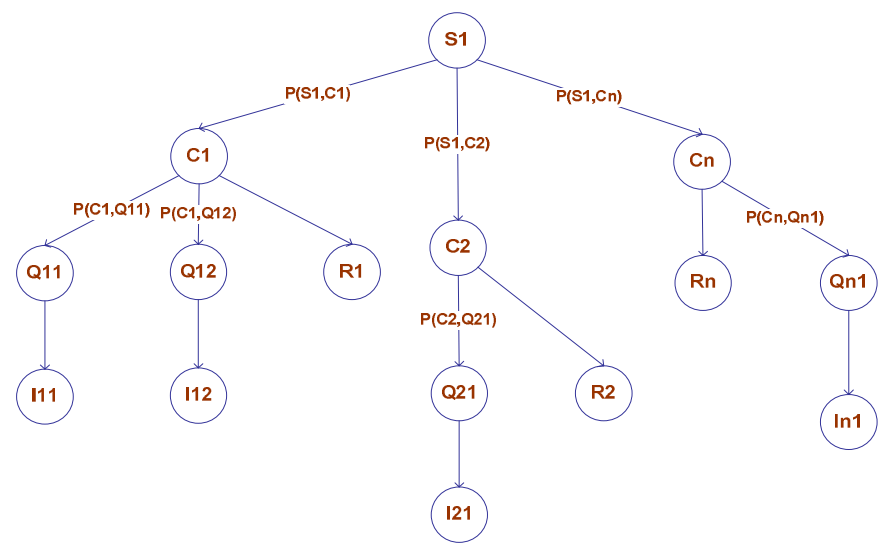

Fig. 2. Risk assessment based on probabilities

Fig. 2 shows the process of calculating the probabilities of consequences associated to the detection of a specific symptom (S). The symptom is the entity that enables the detection of some abnormal situation. Note that it is not the cause of the situation nor the situation it self. Thus the symptom may be caused by one or more possible causes (C). Each cause has one or more consequences $(\mathrm{Q})$. For the sake of simplicity we establish that the measurement of impact I is associated with the consequences $\mathrm{Q}$. The 
identification of the situation with the higher level of risk is done by navigation on the tree following the higher probabilities. The calculation of risk is done by using the formula:

$$
R_{i}=P\left(S_{i}, C_{i}\right) \times P\left(C_{i}, Q_{i j}\right) * I_{i j}
$$

For each combination the method computes a value of risk. The combination with the higher risk value is the one that must be considered, i.e. treated, in first place. Thus if we only considerer the higher risk value than:

$$
R=\max \left\{R_{i}\right\}
$$

Depending on the options made at the set-up phase there is also the possibility of presenting the several values of risks computed for the other consequences in order to guide the user on solving each of the associated causes [7]. This is an option for each company to decide since it is highly dependent on company policies.

Example. Let us consider two problems $P_{1}, P_{2}$ that are stored in the system, and classified as solved problems (i.e. the cause for each of them is already found and the problem was efficiently eliminated). For each one we also have information on "frequency" $(f)$ and "Probable Cause" $\left(P C_{i}\right)$. Now, Let us consider a new third problem $P_{3}$ which has just occurred and about which we only know that has the same symptoms of $P_{1}, P_{2}$. Assuming that: $f_{1}>f_{2}$.

\begin{tabular}{llllll}
\hline$P_{1}$ & & $P_{2}$ & & $P_{3}$ & \\
\hline$f$ & $f_{1}$ & $f$ & $f_{2}$ & $f$ & 1 \\
\hline$P C_{i}$ & $P C_{1}$ & $P C_{i}$ & $P C_{3}$ & & \\
\hline$P C_{i}$ & $P C_{2}$ & $P C_{i}$ & $P C_{4}$ & & \\
\hline
\end{tabular}

Lets say that for problem $P_{1}$ we are pretty sure about its cause but in what concerns $P_{2}$ the possible causes are equally probable. Thus when defining the probabilities we must reflect these considerations, admitting that $p_{1}>p_{2}$ and $p_{1}+p_{2}=1$ and $p_{3}=0.5$.

\begin{tabular}{llll}
\hline$P_{1}$ & & $P_{2}$ & \\
\hline$p\left(P C_{1}\right)$ & $p_{1}$ & $p\left(P C_{3}\right)$ & $p_{3}$ \\
\hline$p\left(P C_{2}\right)$ & $p_{2}$ & $p\left(P C_{4}\right)$ & $p_{3}$ \\
\hline
\end{tabular}

Note that $p\left(P C_{i}\right)=P\left(S_{i}, C_{i}\right)$.

Lets now associate the "Cause" $\left(C_{i}\right)$ to each Probable Cause, assuming that two probable causes are associated to the same cause. This must be considered when computing the probable causes for the new problem.

\begin{tabular}{ll}
\hline$P C_{i}$ & $C_{i}$ \\
\hline$P C_{1}$ & $C_{1}$ \\
\hline$P C_{2}$ & $C_{2}$ \\
\hline$P C_{3}$ & $C_{3}$ \\
\hline$P C_{4}$ & $C_{4}$ \\
\hline
\end{tabular}

Finally we have to define the probability of "Cause" to degenerate in a specific "Consequence" $\left(Q_{i j}\right)$ with an associated impact $\left(I_{i j}\right)$. Assuming that: $p_{4}+p_{5}+p_{6}=1$, $p_{7}+p_{8}=1, p_{9}+p_{10}=1$, and: $p_{4}>p_{5}>p_{6}>p_{7}>p_{8}$ and $p_{10}>p_{9}$. 


\begin{tabular}{ll|ll|ll}
\hline$C_{1}$ & & $C_{2}$ & & $C_{3}$ & \\
\hline$p\left(Q_{11}\right)$ & $p_{4}$ & $p\left(Q_{21}\right)$ & $p_{7}$ & $p\left(Q_{31}\right)$ & $p_{9}$ \\
\hline$p\left(Q_{12}\right)$ & $p_{5}$ & $p\left(Q_{22}\right)$ & $p_{8}$ & $p\left(Q_{32}\right)$ & $p_{10}$ \\
\hline$p\left(Q_{13}\right)$ & $p_{6}$ & & & & \\
\hline
\end{tabular}

Note that $p\left(Q_{i j}\right)=P\left(C_{i}, Q_{i}\right)$.

We are now able of computing the risk associated to this case. Let's start by building the table of the probability of the probable causes that depends o the values established for the frequency:

\begin{tabular}{ll}
\hline$P_{3}$ & \\
\hline$p\left(P C_{1}\right)$ & $f_{1} p_{1}=p_{11}$ \\
\hline$p\left(P C_{2}\right)$ & $f_{1} p_{2}=p_{12}$ \\
\hline$p\left(P C_{3}\right)$ & $f_{2} p_{3}=p_{13}$ \\
\hline$p\left(P C_{4}\right)$ & $f_{2} p_{3}=p_{13}$ \\
\hline
\end{tabular}

The calculated values must be normalised so that the resulting sum is 1 . Note that $p_{11}>p_{12}$. Remember that $P C_{4}$ is related with $C_{1}$, what results in having three different causes, thus we can sum the values $p_{11}$ and $p_{13}$ associated to $p\left(P C_{4}\right)$ and create a new value $p_{14}$.

Let us now build the table of the probability of specific consequence associated to a cause.

\begin{tabular}{ccc}
\hline \multirow{3}{*}{$C_{1}$} & $p\left(Q_{11}\right)$ & $p_{14} p_{4}=p_{15}$ \\
\cline { 2 - 3 } & $p\left(Q_{12}\right)$ & $p_{14} p_{5}=p_{16}$ \\
\cline { 2 - 3 }$C_{2}$ & $p\left(Q_{13}\right)$ & $p_{14} p_{6}=p_{17}$ \\
\cline { 2 - 3 } & $p\left(Q_{21}\right)$ & $p_{12} p_{7}=p_{18}$ \\
\hline \multirow{2}{*}{$C_{3}$} & $p\left(Q_{22}\right)$ & $p_{12} p_{8}=p_{19}$ \\
\cline { 2 - 3 } & $p\left(Q_{31}\right)$ & $p_{13} p_{9}=p_{10}$ \\
\hline
\end{tabular}

Considering the assumptions that we made previously, we have: $\mathrm{p}_{15}>\mathrm{p}_{16}>\mathrm{p}_{17}>\mathrm{p}_{21}>\mathrm{p}_{18}>\mathrm{p}_{20}>\mathrm{p}_{19}$.

At this point we just have to multiply the defined impacts for each consequence, and we will have the different risk values. Note that each consequence $Q_{i j}$ has an associated impact $I_{i j}$. Assuming that: $I_{11}>I_{12}>I_{13}>I_{21}>I_{22}>I_{31}>I_{32}$ we have:

\begin{tabular}{ll}
\hline$R 1$ & $p_{15} I_{11}$ \\
\hline$R 2$ & $p_{16} I_{12}$ \\
\hline$R 3$ & $p_{17} I_{13}$ \\
\hline$R 4$ & $p_{18} I_{21}$ \\
\hline
\end{tabular}

\begin{tabular}{ll}
\hline$R 5$ & $p_{19} I_{22}$ \\
\hline$R 6$ & $p_{20} I_{31}$ \\
\hline$R 7$ & $p_{21} I_{32}$ \\
\hline
\end{tabular}

The selected risk value, following the defined methodology is $R_{l}$ since, considering the conditions imposed, it presents the higher risk value.

Reaching this point we can present the result to the user in terms of what is the most risky situation and which procedure should be applied to avoid it. Remember that we kept tracking of the cause associated to $R_{l}$, thus it is possible to suggest actions that will correct the situation. 
In terms of system we need to keep track of what were the causes involved in the problem evaluation, since even if they were not elected, it is useful to have this information. But, how should this information be stored? Remember that $P_{1}$ and $P_{2}$ have four probable causes but two of them are associated to the same cause, $C_{l}$. Thus to set the probable causes of $P_{3}$ we have to consider that the probability associated to $C_{1}$ is higher that the other two. If we consider the four different probable causes the probability for each of them would be 0.25 . Then, we will have 0.25 for the associated to $C_{2}$ and $C_{3}$ and 0.5 to the associated to $C_{1}$.

\section{Support to Decision}

Through the services developed the users have access to specific functionalities of the system prepared to provide a prompt acquisition and provision of knowledge using an adequate presentation for each type of user. Thus, let us consider again the case shown on Fig. 2 where it would be possible to compute the risk associated to a specific situation. Nevertheless we are able to improve this knowledge with the one that has already been gathered along the life-cycle of the plant. In fact, if we have specific knowledge of the plant it is possible to develop a more accurate and more useful support to decision. For instances if the system stores not only the data that constitutes the case but also the action that was performed to solve it, then it is possible to provide the user with this information which will help him to make a decision on what should be done. In this point of view the support to decision system correlates the past cases that match the current one, and assess the involved risk accordingly. On the top of this strategy each user has to establish what is considered as critical in the considered plant. This information is crucial since it is used to assess the risk for hazardous situations.

Fig. 3 shows the flowchart that describes how the risk is assessed and how this information combined with the information available is used to select the appropriate service. The situation begins while plant is operating normally and a symptom is detected by monitoring systems. After providing the system with a description of the detected symptom, the system will start searching for stored situations that involve symptoms with the same description provided. The stored situations have associated to them one or more symptoms. Each symptom has one or more probable causes (probability) associated and each cause one or more probable consequences (probability). This cascade of information relating the existing knowledge on the situation will be used to compute risk by means of Probabilistic Risk Assessment. Furthermore the existence of knowledge on how to solve the situation, i.e. actions, is also important for the launching of services since they are launched based on this knowledge.

Finally the system will support the user in solving the situation by providing information on which is the adequate service to be used. If the situation is well known, and was not assessed as critical, the system will select "Condition-based maintenance" service. Further the system will suggest the appropriate maintenance action that must be developed based on the information that was already available to cope with that situation. The service of Condition-based maintenance will also support the user to decide when the suggested action should be performed. This scheduling module will also use information of production in order to suggest non producing slots for scheduling the necessary action. 


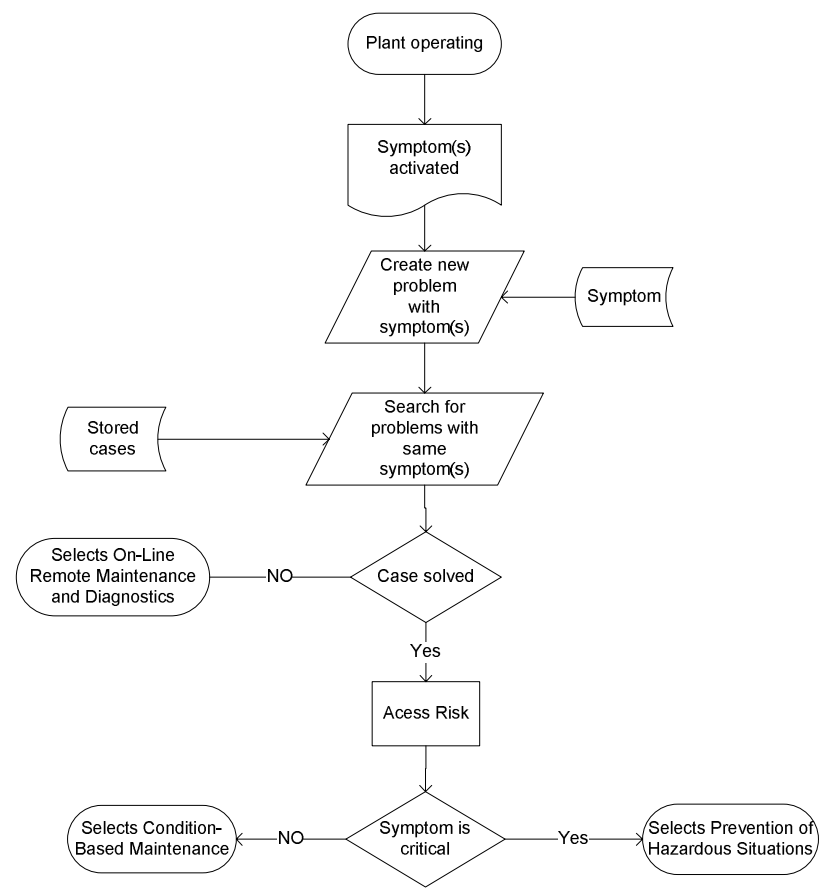

Fig. 3. Decision support using risk assessment

If the situation involves any of the parameters that were previously established as being critical for the plant and their operators, the system will select Prevention of hazardous situations service. Note that the main idea is to prevent the accidents from occurring thus, the actions should be preventive (e.g. operators training). If the situation does not fit in any of the previous categories the system will select Online remote maintenance and diagnostics service. This service aims not only at developing maintenance but also to provide diagnostics to the user. Thus the service uses InLife problem solving module to collect and correlate all the information available that may be related with the situation described. This information is presented to the user in a structured way in order to support him on finding an appropriate solution. If the user was able of finding a solution for the situation, which includes an action to be developed, than the system stores this information and use it if another symptom with the same description is detected or introduced by the user. The calculation of probabilities is highly dependent on this information update since the objective is to use the knowledge acquired during system operation to optimize system performance.

\section{Conclusions and Future Work}

This paper proposes a approach on how to collect information that is scattered along the manufacturing system. This information is then processed and the knowledge derived from it can be used to improve life-cycle management of the industrial plant. With the knowledge acquired it is possible to improve the decision making process by developing 
a set of services specifically focused on its support. The work here presented shows that by assessing the risk associated to the current status of the plant, it is possible to identify the cause for a certain problem and, based on that, propose, actions that support the user on the decision process. The impact of this kind of system is not assessable on a short term basis. Being a life cycle management system, the results need to be measured along time, on a long term approach. Nevertheless the level of structured information that is being gathered and stored and the support to decision offered is seen as very promising for a enhanced life cycle management of the industrial plant.

Future work, will include refinement of the risk assessment algorithm especially in what concerns the interrelation of symptoms and the inclusion of the time effect. Additionally, authors are also interested in exploring the relation of the method with the adaptive systems theory.

\section{References}

1. UNEP/SETAC. Draft Final Report of the LCM Definition Study Version 3.6. Life Cycle Initiative (2003)

2. Raiffa, H.: Decision Analysis: Introductory Lectures on Choices under Uncertainty. Addison-Wesley, Reading (1968)

3. Baker, D., Bridges, D., Hunter, R., Johnson, G., Krupa, J., Murphy, J., Sorenson, K.: Guidebook to Decision-Making Methods. In: WSRC-IM-2002-00002, Department of Energy, USA (2002)

4. Holsapple, C.W.: Decision Support Systems. DSIS Area, School of Management, UK (1999)

5. Marques, M., Neves-Silva, R., Stokic, D., Reimer, P., Agirre, J.: Life-Cycle Management of Complex Manufacturing and Assembly Lines. In: Proceedings of the $14^{\text {th }}$ International Conference on Concurrent Enterprising, Lisbon (2008)

6. Marques, M., Neves-Silva, R.: Risk Assessment to Support Decision on Complex Manufacturing and Assembly Lines. In: Proceeding of the $5^{\text {th }}$ International Conference on Industrial Informatics, Vienna (2007)

7. Marques, M., Neves-Silva, R.: Decision support system using risk assessment for lifecycle management of industrial plants. In: Proceeding of the $13^{\text {th }}$ IFAC Symposium on Information Control Problems in Manufacturing, Moscow (2009)

8. InLife Consortium. InLife Public Concept- Report. InLife Project: Integrated Ambient Intelligence and Knowledge- Based Services for Optimal Life-Cycle Impact of Complex Manufacturing and Assembly Lines, the contract NMP2-CT-2005-517018U (2006)

9. IST Advisory Group. Scenarios for Ambient Intelligence in 2010, IPTS Seville (2001)

10. IST Advisory Group. Ambient Intelligence: from Vision to reality, IST (2003)

11. Bray, D.: Literature Review - Knowledge Management Research at the Organizational Level, Social Science Research Network (2007)

12. Holton, G.A.: Defining Risk. Financial Analysts Journal 60(6), 19-25 (2004)

13. Stamatelatos, M.: Probabilistic Risk Assessment: What Is It And Why Is It Worth Performing It? In: NASA Office of Safety and Mission Assurance (2000)

14. Anderson, K.: Intelligence-Based Threat Assessments for Information Networks and Infrastructures: A White Paper (2005)

15. Ben-Gal, I.: Bayesian Networks. In: Ruggeri, F., Kenett, R., Faltin, F. (eds.) Encyclopedia of Statistics in Quality and Reliability. John Wiley \& Sons, Chichester (2007)

16. Russel, S., Norvig, P.: Artificial Intelligence - A modern Approach. Prentice Hall, Englewood Cliffs (2003) 